\title{
Using Language Modeling to Select Useful Annotation Data
}

\author{
Dmitriy Dligach \\ Department of \\ Computer Science \\ University of Colorado \\ at Boulder \\ Dmitriy.Dligach \\ @colorado.edu
}

\author{
Martha Palmer \\ Department of Linguistics \\ University of Colorado \\ at Boulder \\ Martha.Palmer \\ @colorado.edu
}

\begin{abstract}
An annotation project typically has an abundant supply of unlabeled data that can be drawn from some corpus, but because the labeling process is expensive, it is helpful to pre-screen the pool of the candidate instances based on some criterion of future usefulness. In many cases, that criterion is to improve the presence of the rare classes in the data to be annotated. We propose a novel method for solving this problem and show that it compares favorably to a random sampling baseline and a clustering algorithm.
\end{abstract}

\section{Introduction}

A data set is imbalanced when the distribution of classes in it is dominated by a single class. In Word Sense Disambiguation (WSD), the classes are word senses. The problem of imbalanced data is painfully familiar to WSD researchers: word senses are particularly well known for their skewed distributions that are also highly domain and corpus dependent. Most polysemous words have a sense that occurs in a disproportionately high number of cases and another sense that is seen very infrequently. For example, the OntoNotes (Hovy et al., 2006) sense inventory defines two senses for the verb to add. Of all the instances of this verb in the OntoNotes sense-tagged corpus, $93 \%$ are the instances of the predominant sense (not the arithmetic sense!). Another fact: there are 4,554 total senses in the OntoNotes sense inventory for 1,713 recently released verbs. Only 3,498 of them are present in the actual annotated data. More than 1,000 senses $(23 \%)$ are so rare that they are missing from the corpus altogether. More than a third of the released verbs are missing representative instances of at least one sense. In fact many of the verbs are pseudo-monosemous: even though the sense inventory defines multiple senses, only the most frequent sense is present in the actual annotated data. For example, only 1 out of 8 senses of to rip is present in the data.

The skewed nature of sense distributions is a fact of life. At the same time, a large-scale annotation project like OntoNotes, whose goal is the creation of a comprehensive linguistic resource, cannot simply ignore it. That a sense is rare in a corpus does not mean that it is less important to annotate a sufficient number of instances of that sense: in a different domain it can be more common and not having enough annotated instances of that sense could jeopardize the success of an automatic crossdomain WSD system. For example, sense 8 of to rip ("to import an audio file directly from $\mathrm{CD}$ ") is extremely popular on the web but it does not exist at all in the OntoNotes data. Only the traditional sense of to swap exists in the data but not the computer science sense ("to move a piece of program into memory"), while the latter can conceivably be significantly more popular in technical domains.

In general, class imbalance complicates supervised learning. This contention certainly holds for WSD. As an illustration, consider the verb to call, for which the OntoNotes sense inventory defines 11 senses. Senses 3 and 5 are the most frequent: together they constitute $84 \%$ of the data. To investigate which classes are problematic for a classifi- 
er, we conducted 50 supervised learning experiments. In each experiment one instance of this verb was selected at random and used for testing while the rest was used for training a maximum entropy model. The resulting confusion matrix shows that the model correctly classified most of the instances of the two predominant senses while misclassifying the other classes. The vast majority of the errors came from confusing other senses with sense 5 which is the most frequent sense of to call. Clearly, the data imbalance problem has a significant negative effect on performance.

Let us now envision the following realistic scenario: An annotation project receives funds to sense-tag a set of verbs in a corpus. It may be the case that some annotated data is already available for these verbs and the goal is to improve sense coverage, or no annotated data is available at all. But it turns out there are only enough funds to annotate a portion (e.g. half) of the total instances. The question arises how to pre-select the instances from the corpus in a way that would ensure that all the senses are as well represented as possible. Because some senses of these verbs are very rare, the pool of instances pre-selected for the annotation should include as many as possible instances of the rare senses. Random sampling - the simplest approach - will clearly not work: the pre-selected data will contain roughly the same proportion of the rare sense instances as the original set.

If random sampling is not the answer, the data must be selected in some non-uniform way, i.e. using selective sampling. Active learning (e.g. Chen et al., 2006) is one approach to this problem. Some evidence is available (Zhu and Hovy, 2007) that active learning outperforms random sampling in finding the instances of rare senses. However, active learning has several shortcomings: (1) it requires some annotated data to start the process; (2) it is problematic when the initial training set only contains the data for a single class (e.g. the pseudomonosemous verbs); (3) it is not always efficient in practice: In the OntoNotes project, the data is annotated by two human taggers and the disagreements are adjudicated by the third. In classic active learning a single instance is labeled on each iteration This means the human taggers would have to wait on each other to tag the instance, on the adjudicator for the resolution of a possible disagreement, and finally on the system which still needs to be-retrained to select the next instance to be la- beled, a time sink much greater than tagging additional instances; (4) finally, active learning may not be an option if the data selected needs to be manually pre-processed (e.g. sentence segmented, tokenized, and treebanked - as was the case with some of the OntoNotes data). In this setting, on each iteration of the algorithm, the taggers have to also wait for the selected instance to be manually pre-processed before they can label it.

Thus, it would be significantly more convenient if all the data to be annotated could be pre-selected in advance. In this paper we turn to two unsupervised methods which have the potential to achieve that goal. We propose a simple language modelingbased sampling method (abbreviated as LMS) that increases the likelihood of seeing rare senses in the pre-selected data. The basic approach is as follow: using language modeling we can rank the instances of the ambiguous verb according to their probability of occurrence in the corpus. Because the instances of the rare senses are less frequent than the instances of the predominant sense, we can expect that there will be a higher than usual concentration of the rare sense instances among the instances that have low probabilities. The method is completely unsupervised and the only resource that it requires is a Language Modeling toolkit such as SRILM (Stolcke, 2002), which we used in our experiments. We compare this method with a random sampling baseline and semi-supervised clustering, which can serve the same purpose. We show that our method outperforms both of the competing approaches. We review the relevant literature in section 2, explain the details of LMS in section 3, evaluate LMS in section 4 , discuss the results in section 5 , and describe our plans for future work in section 6 .

\section{Relevant Work}

The problem of imbalanced data has recently received much attention in the machine learning community. Rare classes can be of higher importance than frequent classes, as in medical diagnosis when one is interested in correctly identifying a rare disease. Network intrusion detection faces a similar problem: a malicious activity, although of crucial importance, is a very rare event compared to the large volumes of routine network traffic. At the same time, imbalanced data poses difficulties for an automatic learner in that rare classes have a much higher misclassification rate than common 
ones (Weiss, 1995; Japkowicz, 2001). Learning from imbalanced sets can also be problematic if the data is noisy: given a sufficiently high level of background noise, a learner may not distinguish between true exceptions (i.e. rare cases) and noise (Kubat and Matwin, 1997; Weiss, 2004).

In the realm of supervised learning, costsensitive learning has been recommended as a solution to the problem of learning from imbalanced data (e.g. Weiss, 2004). However, the costs of misclassifying the senses are highly domain specific and hard to estimate. Several studies recently appeared that attempted to apply active learning principles to rare category detection (Pelleg and Moore, 2004; He and Carbonell, 2007). In addition to the issues with active learning outlined in the introduction, the algorithm described in ( $\mathrm{He}$ and Carbonell, 2007) requires the knowledge of the priors, which is hard to obtain for word senses.

WSD has a long history of experiments with unsupervised learning (e.g. Schutze, 1998; Purandare and Peterson, 2004). McCarthy et al. (2004) propose a method for automatically identifying the predominant sense in a given domain. Erk (2006) describes an application of an outlier detection algorithm to the task of identifying the instances of unknown senses. Our task differs from the latter two works in that it is aimed at finding the instances of the rare senses.

Finally, the idea of LMS is similar to the techniques for sentence selection based on rare n-gram co-occurrences used in machine translation (Eck et al., 2005) and syntactic parsing (Hwa, 2004).

\section{Language Modeling for Data Selection}

Our method is outlined in Figure 1:

\begin{tabular}{l}
\hline Input \\
A large corpus that contains $T$ candidate instances \\
from which $S$ instances are to be selected for anno- \\
tation \\
\hline Basic Steps \\
1. Compute the language model for the corpus \\
2. Compute the probability distribution over the $T$ \\
candidate instances of the target verb \\
3. Rank the $T$ candidate instances by their proba- \\
bilities \\
4. Form a cluster by selecting $S$ instances with the \\
lowest probability
\end{tabular}

Figure 1. Basic steps of LMS
Let us now clarify a few practical points. Although an instance of the target verb can be represented as the entire sentence containing the verb, from the experiments with automatic WSD (e.g. Dligach and Palmer, 2008), it is known that having access to just a few words in the neighborhood of the target verb is sufficient in many cases to predict the sense. For the purpose of LMS we represent an instance as the chunk of text centered upon the target verb plus the surrounding words on both sides within a three-word window. Although the size of the window around the target verb is fixed, the actual number of words in each chunk may vary when the target verb is close to the beginning or the end of sentence. Therefore, we need some form of length normalization. We normalize the log probability of each chunk by the actual number of words to make sure we do not favor shorter chunks (SRILM operates in log space). The resulting metric is related to perplexity: for a sequence of words $W=w_{1} w_{2} \ldots w_{N}$ the perplexity is

$$
\begin{aligned}
& P P(W)=P\left(w_{1} w_{2} \ldots w_{N}\right)^{-\frac{1}{N}} \\
& \text { The } \log \text { of perplexity is } \\
& \log [P P(W)]=-\frac{1}{N} \log \left[P\left(w_{1} w_{2} \ldots w_{N}\right)\right]
\end{aligned}
$$

Thus, the quantity we use for ranking is negative perplexity.

\section{Evaluation}

For the evaluation, we selected two-sense verbs from the OntoNotes data that have at least 100 instances and where the share of the rare sense is less than $20 \%$. There were 11 such verbs $(2,230$ instances total) with the average share of the rare sense $11 \%$.

Our task consists of clustering the instances of a verb into two clusters, one of which is expected to have a higher concentration of the rare senses than the other. Since the rare sense cluster is of primary interest to us, we report two metrics: (1) precision: the ratio of the number of instances of the rare sense in the cluster and the total number of instances in the cluster; (2) recall: the ratio of the number of instances of the rare sense in the cluster and the total number of the rare sense instances in both clusters. Note that precision is not of primary importance for this task because the goal is not to reliably identify the instances of the rare sense but 
rather to group them into a cluster where the rare senses will have a higher concentration than in the original set of the candidate instances. At the same time achieving high recall is important since we want to ensure that most, if not all, of the rare senses that were present among the candidate instances are captured in the rare sense cluster.

\subsection{Plausibility of LMS}

The goal of our first set of experiments is to illustrate the plausibility of LMS. Due to space constraints, we examine only two verbs: compare and $a d d$. The remaining experiments will focus on a more comprehensive evaluation that will involve all 11 verbs. We computed the normalized log probability for each instance of a verb. We then ordered these candidate instances by their normalized log probability and computed the recall of the rare sense at various levels of the size of the rare sense cluster. We express the size of the rare sense cluster as a share of the total number of instances. We depict recall vs. cluster size with a dotted curve. The graphs are in Figures 2 and 3.

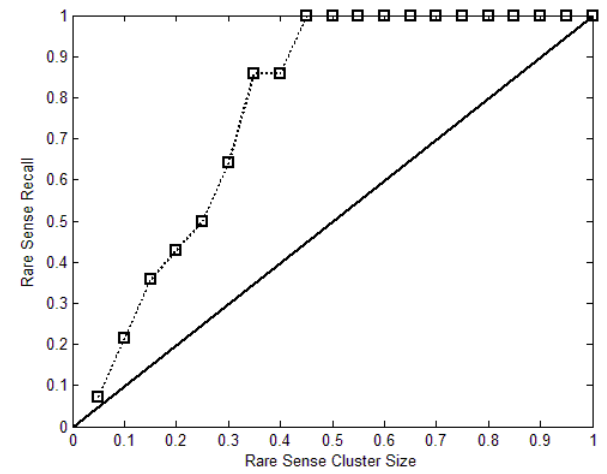

Figure 2. Rare sense recall for compare

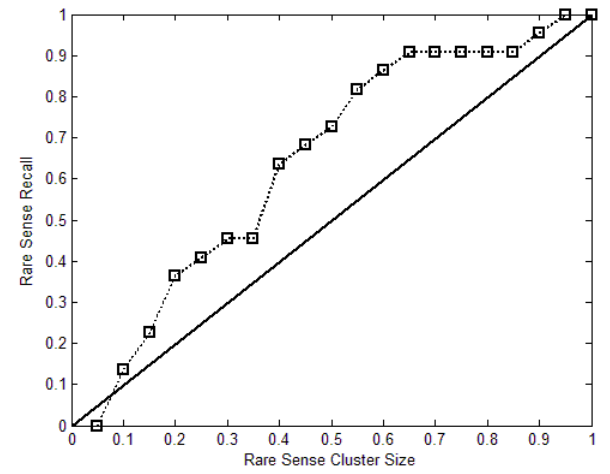

Figure 3. Rare sense recall for add

The diagonal line on these figures corresponds to the random sampling baseline. A successful
LMS would correspond to the dotted curve lying above the random sampling baseline, which happens to be the case for both of these verbs. For compare we can capture all of the rare sense instances in a cluster containing less than half of the candidate instances. While verbs like compare reflect the best-case scenario, the technique we proposed still works for the other verbs although not always as well. For example, for add we can recall more than $70 \%$ of the rare sense instances in a cluster that contains only half of all instances. This is more than 20 percentage points better than the random sampling baseline where the recall of the rare sense instances would be approximately $50 \%$.

\subsection{LMS vs. Random Sampling Baseline}

In this experiment we evaluated the performance of LMS for all 11 verbs. For each verb, we ranked the instances by their normalized log probability and placed the bottom half in the rare sense cluster. The results are in Table 2. The second column shows the share of the rare sense instances in the entire corpus for each verb. Thus, it represents the precision that would be obtained by random sampling. The recall for random sampling in this setting would be 0.5 .

Ten verbs outperformed the random sampling baseline both with respect to precision and recall (although recall is much more important for this task) and one verb performed as well. On average these verbs showed a recall figure that was 22 percentage points better than random sampling. Two of the 11 verbs (compare and point) were able to recall all of the rare sense instances.

\begin{tabular}{llll}
\hline Verb & Rare Inst & Precision & Recall \\
\hline account & 0.12 & 0.21 & 0.93 \\
add & 0.07 & 0.10 & 0.73 \\
admit & 0.18 & 0.18 & 0.50 \\
allow & 0.06 & 0.07 & 0.62 \\
compare & 0.08 & 0.16 & 1.00 \\
explain & 0.10 & 0.12 & 0.60 \\
maintain & 0.11 & 0.11 & 0.53 \\
point & 0.15 & 0.29 & 1.00 \\
receive & 0.07 & 0.08 & 0.60 \\
remain & 0.15 & 0.20 & 0.65 \\
worry & 0.15 & 0.22 & 0.73 \\
\hline average & 0.11 & 0.16 & 0.72 \\
\hline
\end{tabular}

Table 2. LMS results for 11 verbs 


\subsection{LMS vs. K-means Clustering}

Since LMS is a form of clustering one way to evaluate its performance is by comparing it with an established clustering algorithm such as K-means (Hastie et al., 2001). There are several issues related to this evaluation. First, K-means produces clusters and which cluster represents which class is a moot question. Since for the purpose of the evaluation we need to know which cluster is most closely associated with a rare sense, we turn Kmeans into a semi-supervised algorithm by seeding the clusters. This puts LMS at a slight disadvantage since LMS is a completely unsupervised algorithm, while the new version of $\mathrm{K}$-means will require an annotated instance of each sense. However, this disadvantage is not very significant: in a real-world application, the examples from a dictionary can be used to seed the clusters. For the purpose of this experiment, we simulated the examples from a dictionary by simply taking the seeds from the pool of the annotated instances we identified for the evaluation. K-means is known to be highly sensitive to the choice of the initial seeds. Therefore, to make the comparison fair, we perform the clustering ten times and pick the seeds at random for each iteration. The results are averaged.

Second, K-means generates clusters of a fixed size while the size of the LMS-produced clusters can be easily varied. This advantage of the LMS method has to be sacrificed to compare its performance to K-means. We compare LMS to K-means by counting the number of instances that K-means placed in the cluster that represents the rare sense and selecting the same number of instances that have the lowest normalized probability. Thus, we end up with the two methods producing clusters of the same size (with k-means dictating the cluster size).

Third, K-means operates on vectors and therefore the instances of the target verb need to be represented as vectors. We replicate lexical, syntactic, and semantic features from a verb sense disambiguation system that showed state-of-the-art performance on the OntoNotes data (Dligach and Palmer, 2008).

The results of the performance comparison are shown in Table 3. The fourth column shows the relative size of the K-means cluster that was seeded with the rare sense. Therefore it also de- fines the share of the instances with the lowest normalized log probability that are to be included in the LMS-produced rare sense clusters. On average, LMS showed 3\% better recall than K-means clustering.

\begin{tabular}{llllll}
\hline & \multicolumn{3}{c}{ K-means } & \multicolumn{2}{c}{ LMS } \\
\hline verb & precision & recall & size & precision & recall \\
account & 0.21 & 1.00 & 0.58 & 0.20 & 1.00 \\
add & 0.06 & 0.54 & 0.50 & 0.10 & 0.73 \\
admit & 0.21 & 0.31 & 0.29 & 0.09 & 0.15 \\
allow & 0.08 & 0.36 & 0.31 & 0.06 & 0.31 \\
compare & 0.22 & 0.42 & 0.18 & 0.19 & 0.43 \\
explain & 0.16 & 0.61 & 0.44 & 0.14 & 0.60 \\
maintain & 0.13 & 0.91 & 0.80 & 0.11 & 0.82 \\
point & 0.27 & 0.66 & 0.42 & 0.31 & 0.89 \\
receive & 0.11 & 0.68 & 0.72 & 0.08 & 0.80 \\
remain & 0.10 & 0.41 & 0.44 & 0.21 & 0.61 \\
worry & 0.81 & 0.51 & 0.13 & 0.38 & 0.33 \\
\hline average & 0.21 & 0.58 & 0.44 & 0.17 & 0.61 \\
\hline
\end{tabular}

Table 3. LMS vs. K-means

\section{Discussion and Conclusion}

In this paper we proposed a novel method we termed LMS for pre-selecting instances for annotation. This method is based on computing the probability distribution over the instances and selecting the ones that have the lowest probability. The expectation is that instances selected in this fashion will capture more of the instances of the rare classes than would have been captured by random sampling. We evaluated LMS by comparing it to random sampling and showed that LMS outperforms it. We also demonstrated that LMS compares favorably to K-means clustering. This is despite the fact that the cluster sizes were dictated by $\mathrm{K}$-means and that $\mathrm{K}$-means had at its disposal much richer linguistic representations and some annotated data.

Thus, we conclude that LMS is a promising method for data selection. It is simple to use since one only needs the basic functionality that any language modeling toolkit offers. It is flexible in that the number of the instances to be selected can be specified by the user, unlike, for example, when clustering using k-means. 


\section{Future Work}

First, we would like to investigate the effect of selective sampling methods (including LMS) on the performance of WSD models learned from the selected data. Next, we plan to apply LMS for Domain adaptation. Unlike the scenario we dealt with in this paper, the language model would have to be learned from and applied to different corpora: it would be trained on the source corpus and used to compute probabilities for the instances in the target corpus that needs to be adapted. We will also experiment with various outlier detection techniques to determine their applicability to data selection. Another promising direction is a simplified active learning approach in which a classifier is trained on the labeled data and applied to unlabeled data; the instances with a low classifier's confidence are selected for annotation (i.e. this is active learning conducted over a single iteration). This approach is more practical than the standard active learning for the reasons mentioned in Section 1 and should be compared to LMS. Finally, we will explore the utility of LMS-selected data as the initial training set for active learning (especially in the cases of the pseudo-monosemous verbs).

\section{Acknowledgments}

We gratefully acknowledge the support of the National Science Foundation Grant NSF-0715078, Consistent Criteria for Word Sense Disambiguation, and the GALE program of the Defense Advanced Research Projects Agency, Contract No. HR0011-06-C-0022, a subcontract from the BBNAGILE Team. Any opinions, findings, and conclusions or recommendations expressed in this material are those of the authors and do not necessarily reflect the views of the National Science Foundation.

\section{References}

Jinying Chen, Andrew Schein, Lyle Ungar, and Martha Palmer. 2006. An Empirical Study of the Behavior of Active Learning for Word Sense Disambiguation. In Proceedings of the HLT-NAACL.

Dmitriy Dligach and Martha Palmer. 2008. Novel Semantic Features for Verb Sense Disambiguation. In Proceedings of ACL-HLT.

Matthias Eck, Stephan Vogel, and Alex Waibel. 2005. Low Cost Portability for Statistical Machine Transla- tion Based on N-gram Frequency and TF-IDF. Proceedings of IWSLT 2005.

Katrin Erk. Unknown Word Sense Detection as Outlier Detection. 2006. In Proceedings of HLT-NAACL.

Trevor Hastie, Robert Tibshirani, and Jerome Friedman. The Elements of Statistical Learning. Data Mining, Inference, and Prediction. 2001. Springer.

Jingrui $\mathrm{He}$ and Jaime Carbonell. 2007. NearestNeighbor-Based Active Learning for Rare Category Detection. NIPS.

Hovy, E.H., M. Marcus, M. Palmer, S. Pradhan, L. Ramshaw, and R. Weischedel. 2006. OntoNotes: The 90\% Solution. In Proceedings of the HLT-NAACL.

Eduard Hovy and Jingbo Zhu. 2007. Active Learning for Word Sense Disambiguation with Methods for Addressing the Class Imbalance Problem. In Proceedings of EMNLP.

Rebecca Hwa. 2004. Sample Selection for Statistical Parsing. Computational Linguistics. Volume 30. Issue 3.

Natalie Japkowicz. 2001. Concept Learning in the Presence of Between-Class and Within-Class Imbalances. Proceedings of the Fourteenth Conference of the Canadian Society for Computational Studies of Intelligence, Springer-Verlag.

Miroslav Kubat and Stan Matwin. 1997. Addressing the curse of imbalanced training sets: one-sided selection. In Proceedings of the Fourteenth International Conference on Machine Learning.

Diana McCarthy, Rob Koeling, Julie Weeds, and John Carroll. 2004. Finding Predominant Word Senses in Untagged Text. In Proceedings of 42nd Annual Meeting of Association for Computational Linguistics.

Dan Pelleg and Andrew Moore. 2004. Active Learning for Anomaly and Rare-Category Detection. NIPS.

Amruta Purandare and Ted Pedersen. Word Sense Discrimination by Clustering Contexts in Vector and Similarity Spaces. 2004. In Proceedings of the Conference on CoNLL.

Hinrich Schutze. 1998 Automatic Word Sense Discrimination. Computational Linguistics.

Andreas Stolcke. 2002. SRILM - An Extensible Language Modeling Toolkit. In Proc. Intl. Conf. Spoken Language Processing, Denver, Colorado.

Gary M. Weiss. 1995. Learning with Rare Cases and Small Disjuncts. Proceedings of the Twelfth International Conference on Machine Learning, Morgan Kaufmann.

Gary M. Weiss. 2004. Mining with Rarity: A Unifying Framework. SIGKDD Explorations, special issue on learning from imbalanced datasets. 